\title{
Vibrations of String due to a Uniform Partially Distributed Moving Load: Closed Solutions
}

\author{
Rafał Idzikowski, ${ }^{1}$ Małgorzata Mazij, ${ }^{1}$ Paweł Śniady, ${ }^{1}$ and Piotr Śniady ${ }^{2,3}$ \\ ${ }^{1}$ Faculty of Environmental Engineering and Geodesy, Wrocław University of Environmental and Life Science, \\ Plac Grunwaldzki 24, 50-363 Wrocław, Poland \\ ${ }^{2}$ Instytut Matematyczny, Polska Akademia Nauk, Ulica Śniadeckich 8, 00-956 Warszawa, Poland \\ ${ }^{3}$ Instytut Matematyczny, Uniwersytet Wrocławski, Plac Grunwaldzki 2/4, 50-384 Wrocław, Poland \\ Correspondence should be addressed to Paweł Śniady; pawel.sniady@wp.pl
}

Received 22 May 2013; Accepted 23 October 2013

Academic Editor: Xiaojun Wang

Copyright ( 2013 Rafał Idzikowski et al. This is an open access article distributed under the Creative Commons Attribution License, which permits unrestricted use, distribution, and reproduction in any medium, provided the original work is properly cited.

\begin{abstract}
We consider the damping dynamical response of a finite string due to a uniformly distributed load and a point force moving with a constant velocity. The classical solution for the transverse displacement of a string has a form of a sum of two infinite series, one of which represents the forced vibrations (aperiodic vibrations) and the other one represents free vibrations of the beam. We show that the series which represents aperiodic (forced) vibrations of the string can be presented in a closed, analytical form.
\end{abstract}

\section{Introduction}

The moving load problem (which is one of the most important problems in the dynamics of structures) has been studied by many authors for many years. This problem occurs in dynamics of bridges, roadways, railways, and runways as well as missiles and aircrafts. Different types of structures and girders like beams, plates, shells, and frames have been considered. Also different models of moving loads have been assumed [1]. In the paper, we study damping vibration of a finite string subject to a moving load. A string as a simple model of a one-dimensional continuous system resistant to tension but not to bending is often used in analysis of numerous engineering structures and has been a subject of great scientific interest for a considerable time. This follows from the fact that the vibrations of a string are described by the wave differential equation. This allows one to see the wave effect in a string, contrary to many more complex systems where it might be either not present or not clearly visible. The analogies between a string and the beams have been considered in papers [2-4]. Various aspects of the dynamics response of a string under a moving load have been considered, among others, in the papers [5-14]. The classical solution of the response of a finite string subjected to a load moving with a constant velocity has a form of an infinite series. The main goal of this paper is to show that, in the case when the finite string is loaded by an uniformly distributed load and also a point force moving with a constant velocity, the aperiodic part of the solution can be presented in a closed form instead of an infinite series. Using the method, of superposed deflections Kaczkowski [15] has shown for a simply supported Euler-Bernoulli beam that, in the case of undamped vibration, the aperiodic part of the solution can be presented in a closed form. Next, Reipert obtained a closed form solutions for a beam with arbitrary boundary conditions [16] and for a frame [17]. In this paper, we use a different method to obtain the solutions in a closed form. The presented method of finding a solution in a closed form is based on the observation that the solution of some partial differential equation in the form of an infinite series is also a solution of an appropriate ordinary differential equation. The solutions of the ordinary equation can be presented in the closed form. The presented solutions can be also used in axial and torsional vibration of the rod. Using this method, the closed solutions for undamped vibration of string and Timoshenko beam due to moving point force have been obtained in the papers $[13,18]$. 


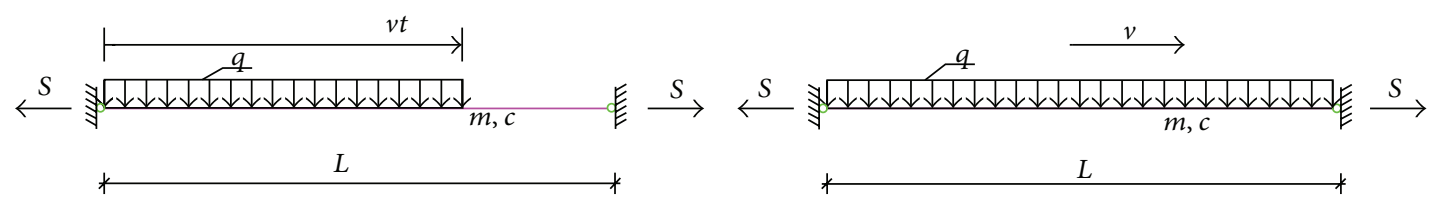

(a)

(b)

FIGURE 1: Scheme of string vibration due to moving load.

\section{Damped Vibrations}

We consider damping vibrations of a string of finite length $L$ subjected to a uniformly partially distributed load moving with a constant velocity $v$ (Figure $1(\mathrm{a})$ ). Vibrations of the string are described by

$$
-S \frac{\partial^{2} w(x, t)}{\partial x^{2}}+c \frac{\partial w(x, t)}{\partial t}+m \frac{\partial^{2} w(x, t)}{\partial t^{2}}=p H(v t-x),
$$

where $S$ is the tension of the string, $m$ denotes the mass per unit length of the string, $c$ is damping coefficient, $H(\cdot)$ denotes Heaviside step function, and $p$ is the intensity of the load.

The boundary conditions have the form

$$
w(0, t)=w(L, t)=0 .
$$

After introducing dimensionless variables

$$
\xi=\frac{x}{L}, \quad T=\frac{v t}{L}, \quad \xi \in[0,1], T \in[0,1] .
$$

Equation (1) takes the form

$$
-[w(\xi, T)]^{I I}+c_{0} \dot{w}(\xi, T)+\eta^{2} \ddot{w}(\xi, T)=p_{0} H(T-\xi),
$$

where $c_{0}=c(v L / S), \eta=v / v_{s}, v_{s}=\sqrt{S / m}, p_{0}=p L^{2} / S$, and $T \leq 1$. The quantity $v_{s}$ represents the wave velocity in the string. The Roman numerals denote differentiation with respect to the spatial coordinate $\xi$, and the dots denote differentiation with respect to the time $T$.

The boundary conditions (2) have the form

$$
w(0, T)=w(1, T)=0 .
$$

Let the initial conditions have the form

$$
w(\xi, 0)=0, \quad \dot{w}(\xi, 0)=0 .
$$

The solution of (4) for boundary conditions (5) is assumed to be in the form of the sine series

$$
w(\xi, T)=\sum_{n=1}^{\infty} y_{n}(T) \sin n \pi \xi .
$$

After substituting the expression (7) into (4) and using the orthogonality method, one obtains the set of ordinary differential equations

$$
\ddot{y}_{n}(T)+2 \alpha \dot{y}_{n}(T)+\omega_{n}^{2} y_{n}(T)=\frac{2 p_{0}}{\eta^{2}} \frac{[1-\cos n \pi T]}{n \pi},
$$

where $\omega_{n}^{2}=(n \pi)^{2} / \eta^{2}, \alpha=c_{0} / 2 \eta^{2}$.
The initial conditions have the form

$$
y_{n}(0)=0, \quad \dot{y}_{n}(0)=0 .
$$

Finally, the response of the string taking into account the solution of (8) and initial conditions (9) can be obtained in the form

$$
w(\xi, T)=w_{A}(\xi, T)+w_{S}(\xi, T)
$$

where

$$
\begin{aligned}
w_{A}(\xi, T)= & w_{A}^{*}(\xi)+w_{A}^{* *}(\xi, T)+w_{A}^{* * *}(\xi, T) \\
= & 2 p_{0} \sum_{n=1}^{\infty} \frac{\sin n \pi \xi}{(n \pi)^{3}} \\
& -4 p_{0} \alpha \eta^{2} \sum_{n=1}^{\infty} \frac{\sin n \pi T \sin n \pi \xi}{\left(1-\eta^{2}\right)^{2}(n \pi)^{4}+4 \alpha^{2} \eta^{4}(n \pi)^{2}} \\
& -2 p_{0}\left(1-\eta^{2}\right) \sum_{n=1}^{\infty} \frac{n \pi \cos n \pi T \sin n \pi \xi}{\left(1-\eta^{2}\right)^{2}(n \pi)^{4}+4 \alpha^{2} \eta^{4}(n \pi)^{2}}
\end{aligned}
$$

$$
\begin{aligned}
& w_{S}=2 p_{0} \alpha e^{-\alpha T} \\
& \times \sum_{n=1}^{\infty}\left[-\frac{1}{(n \pi)^{3} \Omega_{n}}\right. \\
& \left.+\frac{\left(1+\eta^{2}\right)}{\Omega_{n}(n \pi)\left[\left(1-\eta^{2}\right)^{2}(n \pi)^{2}+4 \alpha^{2} \eta^{4}\right]}\right] \\
& \times \sin \Omega_{n} T \sin n \pi \xi+2 p_{0} e^{-\alpha T} \\
& \times \sum_{n=1}^{\infty}\left[-\frac{1}{(n \pi)^{3}}\right. \\
& \left.+\frac{\left(1-\eta^{2}\right)}{(n \pi)\left[\left(1-\eta^{2}\right)^{2}(n \pi)^{2}+4 \alpha^{2} \eta^{4}\right]}\right] \\
& \times \cos \Omega_{n} T \sin n \pi \xi
\end{aligned}
$$

where

$$
\Omega_{n}^{2}=\left(\frac{n \pi}{\eta}\right)^{2}-\alpha^{2}
$$


The aperiodic vibrations of the string can be presented in closed form. Let us assume that $\eta \neq 1$. The first function in (11) is equal to

$$
w_{A}^{*}(\xi)=2 p_{0} \sum_{n=1}^{\infty} \frac{\sin n \pi \xi}{(n \pi)^{3}}=p_{0}\left[\frac{1}{6} \xi^{3}-\frac{1}{2} \xi^{2}+\frac{1}{3} \xi\right] .
$$

Let us notice that the function

$$
w_{A}^{* *}(\xi, T)=-4 p_{0} \alpha \eta^{2} \sum_{n=1}^{\infty} \frac{\sin n \pi T \sin n \pi \xi}{\left(1-\eta^{2}\right)^{2}(n \pi)^{4}+4 \alpha^{2} \eta^{4}(n \pi)^{2}}
$$

is a solution of the ordinary differential equation (see [13])

$$
\begin{gathered}
\frac{d^{4} w_{A}^{* *}(\xi, T)}{d \xi^{4}}-4\left(\frac{\alpha \eta^{2}}{1-\eta^{2}}\right)^{2} \frac{d^{2} w_{A}^{* *}(\xi, T)}{d \xi^{2}} \\
=-\frac{2 p_{0} \alpha \eta^{2}}{\left(1-\eta^{2}\right)^{2}} \delta(\xi-T)
\end{gathered}
$$

for the boundary conditions

$$
\begin{aligned}
w_{A}^{* *}(0, T) & =w_{A}^{* *}(1, T)=0 \\
\left.\frac{\partial^{2} w_{A}^{* *}(\xi, T)}{\partial \xi^{2}}\right|_{\xi=0} & =\left.\frac{\partial^{2} w_{A}^{* *}(\xi, T)}{\partial \xi^{2}}\right|_{\xi=1}=0 .
\end{aligned}
$$

After solving (16) using the Laplace transform, we can obtain the function $w_{A}^{* *}(\xi, T)$ in the closed form instead of a series. This closed solution has the form

$$
\begin{aligned}
& w_{A}^{* *}(\xi, T)=-\frac{p_{0}}{\left(1-\eta^{2}\right) a} \\
& \times\left[\xi(1-T)-\frac{\sinh a \xi \sinh a(1-T)}{a \sinh a}\right] \\
& \text { for } \xi \leq T, \\
& w_{A}^{* *}(\xi, T)=-\frac{p_{0}}{\left(1-\eta^{2}\right) a} \quad \text { for } \xi \geq T, \\
& \times\left[(1-\xi) T-\frac{\sinh a(1-\xi) \sinh a T}{a \sinh a}\right]
\end{aligned}
$$

where $a=2\left(\alpha \eta^{2} /\left(1-\eta^{2}\right)\right)$.

The function

$$
w_{A}^{* * *}(\xi, T)=-2 p_{0} \sum_{n=1}^{\infty} \frac{\left(1-\eta^{2}\right) n \pi \cos n \pi T \sin n \pi \xi}{\left(1-\eta^{2}\right)^{2}(n \pi)^{4}+4 \alpha^{2} \eta^{4}(n \pi)^{2}}
$$

is a solution of the ordinary differential equation

$$
\begin{aligned}
& \frac{d^{4} w_{A}^{* * *}(\xi, T)}{d \xi^{4}}-4\left(\frac{\alpha \eta^{2}}{1-\eta^{2}}\right)^{2} \\
& \quad \times \frac{d^{2} w_{A}^{* * *}(\xi, T)}{d \xi^{2}}=\frac{p_{0}}{\left(1-\eta^{2}\right)} \frac{d \delta(\xi-T)}{d \xi},
\end{aligned}
$$

for the boundary conditions similar to (17).
The closed solution of the function $w_{A}^{* *}(\xi, T)$ has the form

$$
\begin{aligned}
w_{A}^{* * *}(\xi, T)= & \frac{p_{0}}{\left(1-\eta^{2}\right) a^{2}} \\
& \times\left[\xi-\frac{\sinh a \xi \cosh a(1-T)}{\sinh a}\right] \\
\text { for } \xi \leq T, & \\
w_{A}^{* * *}(\xi, T)= & \frac{p_{0}}{\left(1-\eta^{2}\right) a^{2}} \\
& \times\left[-(1-\xi)+\frac{\sinh a(1-\xi) \cosh a T}{\sinh a}\right] \\
& \text { for } \xi \geq T .
\end{aligned}
$$

Let us consider the situation when $T \geq 1$ (Figure 1(b)). In this case, the solution has the form

$$
w_{A}(\xi)=2 p_{0} \sum_{n=1}^{\infty} \frac{1-(-1)^{n}}{(n \pi)^{3}} \sin n \pi \xi=\frac{1}{2} p_{0} \xi(1-\xi) .
$$

The general integral can be presented in the form

$$
w_{S}(\xi, T)=w_{S 1}(\xi, T)+w_{S 2}(\xi, T-1),
$$

where function $w_{S 1}(\xi, T)$ is given by expression (12) assuming $T \geq 1$. The function $w_{S 2}(\xi, T-1)$ has the form

$$
\begin{aligned}
w_{s}(\xi, T)= & 2 p_{0} e^{-\alpha(T-1)} \\
& \times \sum_{n=1}^{\infty}\left[A_{n} \sin \Omega_{n}(T-1)+B_{n} \cos \Omega_{n}(T-1)\right] \\
& \times \sin n \pi \xi,
\end{aligned}
$$

where

$$
\begin{gathered}
A_{n}=-\frac{\alpha}{\Omega_{n}} \frac{\left[1-(-1)^{n}\right]}{(n \pi)^{3}}, \\
B_{n}=-\frac{\left[1-(-1)^{n}\right]}{(n \pi)^{3}} .
\end{gathered}
$$

\section{Undamped Vibrations}

Let us consider undamped vibration of a string due to a uniformly partially distributed load moving with a constant velocity. Assuming $c=0(\alpha=0)$, the solution (10)-(12) has the form

$$
w(\xi, T)=w_{A}(\xi, T)+w_{S}(\xi, T),
$$

where

$$
\begin{aligned}
w_{A}(\xi, T)= & w_{A 1}(\xi, T)+w_{A 2}(\xi, T) \\
= & 2 p_{0} \sum_{n=1}^{\infty} \frac{\sin n \pi \xi}{(n \pi)^{3}}-\frac{2 p_{0}}{\left(1-\eta /^{2}\right)} \\
& \times \sum_{n=1}^{\infty} \frac{\cos n \pi T \sin n \pi \xi}{(n \pi)^{3}}
\end{aligned}
$$




$$
\begin{aligned}
= & p_{0}\left[\frac{1}{6} \xi^{3}-\frac{1}{2} \xi^{2}+\frac{1}{3} \xi\right] \\
& -\frac{2 p_{0}}{\left(1-\eta^{2}\right)} \sum_{n=1}^{\infty} \frac{\cos n \pi T \sin n \pi \xi}{(n \pi)^{3}}, \\
w_{S}= & \frac{2 p_{0} \eta^{2}}{\left(1-\eta^{2}\right)} \sum_{n=1}^{\infty} \frac{\cos (n \pi / \eta) T \sin n \pi \xi}{(n \pi)^{3}} .
\end{aligned}
$$

The function given by the second series in (27) is a solution of the ordinary equation

$$
\frac{d^{4} w_{A 2}(\xi, T)}{d \xi^{4}}=\frac{p_{0}}{1-\eta^{2}} \frac{d \delta(\xi-T)}{d \xi},
$$

for the boundary conditions like (17). After solving (29), one obtains

$$
\begin{aligned}
w_{A 2}(\xi, T)= & -\frac{2 p_{0}}{\left(1-\eta^{2}\right)} \sum_{n=1}^{\infty} \frac{\cos n \pi T \sin n \pi \xi}{(n \pi)^{3}}=\frac{p_{0}}{2\left(1-\eta^{2}\right)} \\
& \times\left[-\frac{\xi^{3}}{3}+\frac{\xi}{3}-(1-T)^{2} \xi+H(\xi-T)(\xi-T)^{2}\right] .
\end{aligned}
$$

Also the function $w_{S}(\xi, T)$ can be presented in a closed form. For the interval $i \eta \leq T \leq(i+1) \eta \leq 1$, where $i=0,2,4, \ldots, n$, the function $w_{S}(\xi, T)$ is also a solution of

$$
\frac{d^{4}\left[w_{S}(\xi, T)\right]}{d \xi^{4}}=-\frac{p_{0} \eta^{2}}{1-\eta^{2}} \frac{d}{d \xi} \delta\left[\xi-\left(\frac{T}{\eta}-i\right)\right]
$$

and hence can be presented in the closed form

$$
\begin{aligned}
w_{s}(\xi, T)=-\frac{p_{0} \eta^{2}}{1-\eta^{2}}\{\xi & +\left[\frac{1}{2}\left(1+i-\frac{T}{\eta}\right)^{2}-1\right] \xi^{3} \\
& \left.-\frac{1}{2}\left(\xi+i-\frac{T}{\eta}\right)^{2} H\left(\xi+i-\frac{T}{\eta}\right)\right\} .
\end{aligned}
$$

For the interval $i \eta \leq T \leq(i+1) \eta \leq 1$, where $i=1,3,5, \ldots, n+$ 1 , the function $w_{I}^{S}(\xi, T)$ is also a solution of

$$
\frac{d^{4}\left[w_{S}(\xi, T)\right]}{d \xi^{4}}=\frac{p_{0} \eta^{2}}{1-\eta^{2}} \frac{d}{d \xi} \delta\left[\xi-\left(i+1-\frac{T}{\eta}\right)\right]
$$

and hence can be presented in the closed form

$$
\begin{aligned}
w_{S}(\xi, T)=\frac{p_{0} \eta^{2}}{2\left(1-\eta^{2}\right)}\{ & {\left[-\left(\frac{T}{\eta}-i\right)^{2}-\frac{1}{3}\right] \xi+\frac{\xi^{3}}{3} } \\
+ & {\left[\xi-(i+1)+\frac{T}{\eta}\right] H } \\
& \left.\times\left[\xi-\left(i+1-\frac{T}{\eta}\right)\right]\right\} .
\end{aligned}
$$

Taking into account the relationships (30), (32), and (34) the function $w(\xi, T)=w_{A}(\xi, T)+w_{S}(\xi, T)$ can be presented in a closed analytical form. For the interval in $\leq T \leq(i+1) \eta \leq 1$, where $i=0,2, \ldots, n$ and $\eta<1\left(v<v_{s}\right)$, one obtains

$$
\begin{aligned}
w(\xi, T)= & \frac{p_{0}}{\left(1-\eta^{2}\right)} \\
& \times\left[\frac{-\eta^{2}}{6} \xi^{3}-\frac{1}{2} \xi^{2}+\frac{1}{2} \xi+\frac{1}{2}(\xi-T)^{3} H(\xi-T)\right] \\
& -\frac{p_{0} \eta^{2}}{1-\eta^{2}}\left\{\xi+\left[\frac{1}{2}\left(1+i-\frac{T}{\eta}\right)^{2}-1\right] \xi^{3}\right. \\
& \left.-\frac{1}{2}\left(\xi+i-\frac{T}{\eta}\right)^{2} H\left(\xi+i-\frac{T}{\eta}\right)\right\},
\end{aligned}
$$

and for the interval $i \eta \leq T \leq(i+1) \eta \leq 1$, where $i=$ $1,3, \ldots, n+1$ and $\eta<1$, one obtains

$$
\begin{aligned}
w_{I}(\xi, T)=\frac{P_{0}}{1-\eta^{2}}\{ & (1-T) \xi+(T-i \eta) \xi \\
& -(\xi-T) H(\xi-T) \\
& -[\eta \xi-\eta(i+1)+T] \\
& \left.\times H\left[\xi-\left(i+1-\frac{T}{\eta}\right)\right]\right\} .
\end{aligned}
$$

Equation (35) gives the string response when the front of the free wave moves in the same direction as the point force while (36) gives the string response when it moves in the opposite direction. For example, for $0 \leq T \leq \eta<1$, it follows from (35) $(i=0)$ that (Figure 2(a))

$$
\begin{aligned}
w(\xi, T)=\frac{p_{0}}{2}\{ & -\xi^{2}+\xi+\frac{1}{1-\eta^{2}} \xi\left[-(1-T)^{2}+(\eta-T)^{2}\right] \\
& +\frac{(\xi-T)^{2}}{1-\eta^{2}} H(\xi-T) \\
& \left.-\frac{(\eta \xi-T)^{2}}{1-\eta^{2}} H\left(\xi-\frac{T}{\eta}\right)\right\} .
\end{aligned}
$$

From (30) or (31) and (33) for $i=0$, we obtain the solution for $\eta>1$, that is, when the load velocity is bigger than the transverse wave velocity (Figure 2(b)); namely,

$$
\begin{aligned}
w(\xi, T)=\frac{p_{0}}{2}\{ & -\xi^{2}+\xi-\frac{1}{\eta^{2}-1} \xi\left[-(1-T)^{2}+(\eta-T)^{2}\right] \\
& +\frac{(\eta \xi-T)^{2}}{\eta^{2}-1} H\left(\xi-\frac{T}{\eta}\right) \\
& \left.-\frac{(\xi-T)^{2}}{\eta^{2}-1} H(\xi-T)\right\} .
\end{aligned}
$$




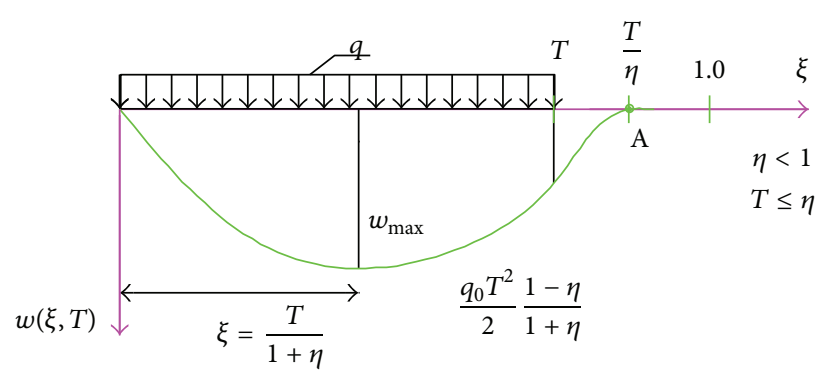

(a)

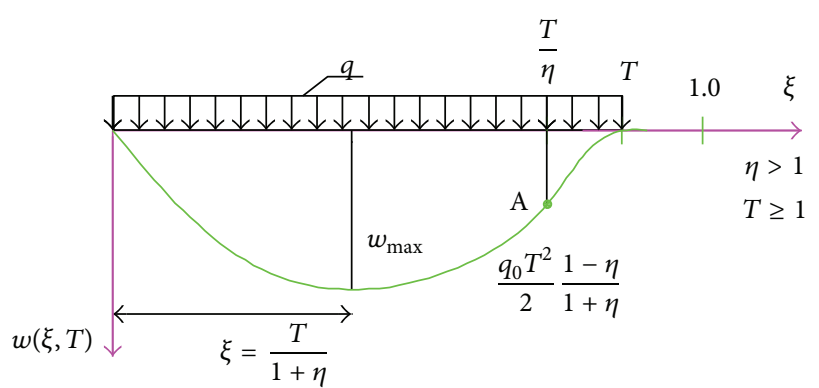

(b)

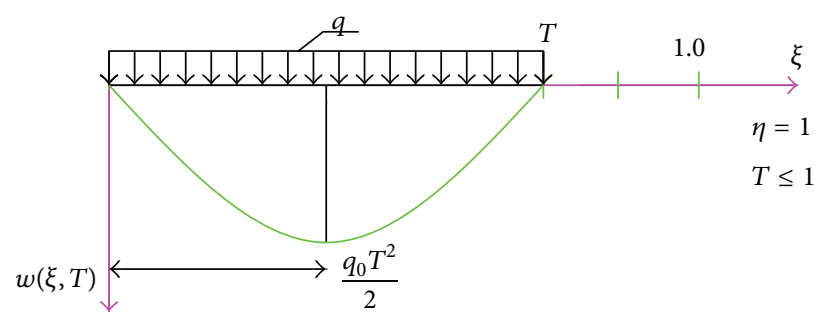

(c)

Figure 2: Transverse displacement of the string due to moving load for $\eta<1, \eta\rangle 1$, and $\eta=1$.

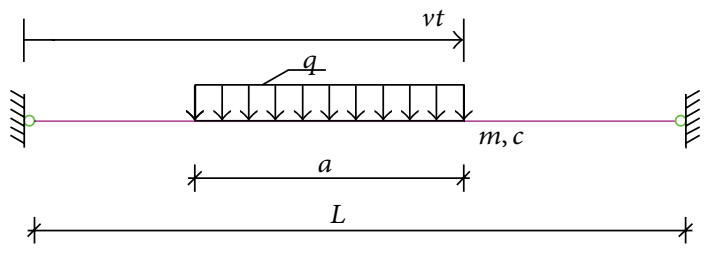

(a)
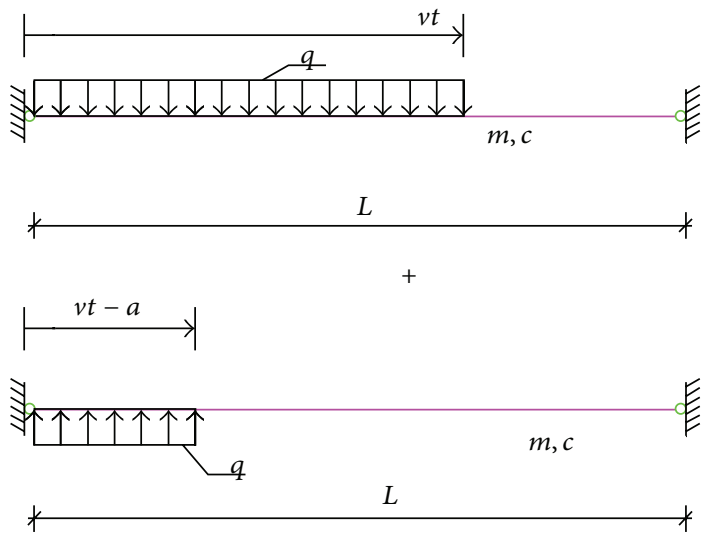

(b)

FiguRE 3: Interval uniformly distributed moving load.

In the particular case when $\eta=1\left(v=v_{s}\right),(37)$ and (38) imply that (Figure 2(c))

$$
\begin{gathered}
w(\xi, T)=\frac{p_{0}}{2}\left(-\xi^{2}+T \xi\right), \quad \text { for } \xi \leq T, \\
w(\xi, T)=0 \quad \text { for } \xi \geq T .
\end{gathered}
$$

The letter A in Figures 2(a) and 2(b) denotes the front of the wave excited by the initial entering of the load to the string.

\section{The Interval of Moving Load}

Let the vibrations of the string be caused by a moving load, uniformly distributed on $a$ (Figure 3(a)).

The solution of the problem can be obtained by superposition of the solutions which are given in Sections 2 and 3 (see Figure 3(b)). One can consider three phases, namely, the entry of the load on the string, the full load and exit of load with string (see Figure 4).

\section{The Inertial Moving Load}

Let us consider the vibrations of the string under uniformly distributed moving mass $m_{1}$,

$$
\begin{array}{r}
-S \frac{\partial^{2} w(x, t)}{\partial x^{2}}+c \frac{\partial w(x, t)}{\partial t}+m \frac{\partial^{2} w(x, t)}{\partial t^{2}} \\
=\left[m_{1} g-m_{1}\left(\frac{\partial^{2} w(x, t)}{\partial t^{2}}+2 v \frac{\partial^{2} w(x, t)}{\partial x \partial t}\right.\right. \\
\left.\left.+v^{2} \frac{\partial^{2} w(x, t)}{\partial x^{2}}\right)\right] H(x-v t) .
\end{array}
$$




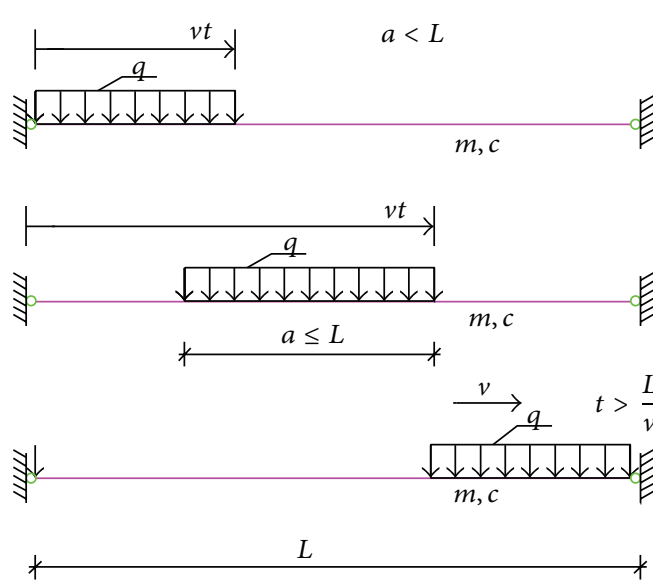

(a)

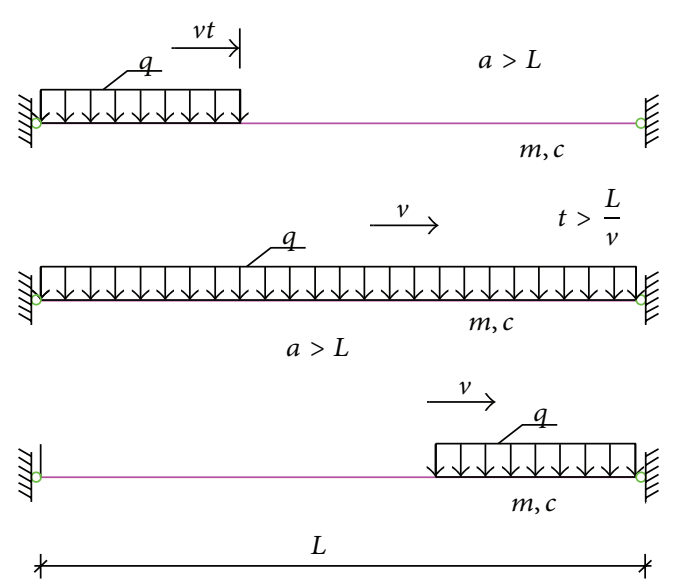

(b)

FIGURE 4: Phases of moving interval uniformly distributed load.

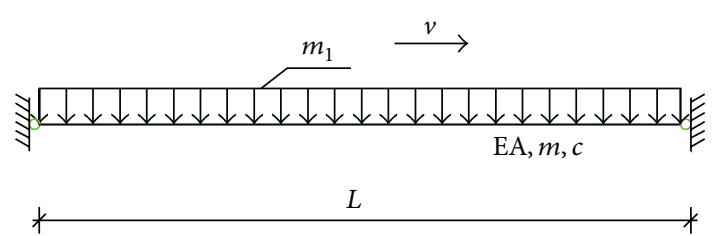

FIGURE 5: Scheme of string under moving uniformly distributed mass.

After using dimensionless variables, (3) and (40), have the form

$$
\begin{aligned}
- & w^{I I}(\xi, T)+c_{0} \dot{w}(\xi, T)+\eta^{2} \ddot{w}(\xi, T) \\
= & \left\{\frac{m_{1} g L^{2}}{S}-\frac{m_{1} v^{2}}{S}\left[\ddot{w}(\xi, T)+2 \dot{w}^{I}(\xi, T)+w^{I I}(\xi, T)\right]\right\} \\
& \times H(\xi-T) .
\end{aligned}
$$

Let us consider only the steady-state solution for $T>1$ (Figure 5). In this case $w(\xi, T)=w_{\text {steady }}(\xi)$ and from (40), one obtains the ordinary equation

$$
-w_{\text {steady }}^{I I}(\xi)\left[1-\left(\frac{v}{v_{c r}}\right)^{2}\right]=\frac{m_{1} g L^{2}}{S},
$$

where the critical velocity of the moving mass is equal to $v_{c r}=$ $\sqrt{S / m_{1}}$. After solving (42), one obtains the displacement of the string in the form

$$
w_{\text {steady }}(\xi)=\frac{g L^{2}}{2\left[v_{c r}^{2}-v^{2}\right]} \xi(1-\xi) .
$$

For $v<v_{c r}$, the displacement of the string is positive, but for $v>v_{c r}$, it is negative. Let us notice that if the velocity is $v<v_{c r}$ and tends to $v_{c r}\left(v \rightarrow v_{c r}\right)$, the response of the string tends to plus infinity, but if $v>v_{c r}$ and tends to $v_{c r}$, the displacement of the string tends to minus infinity.

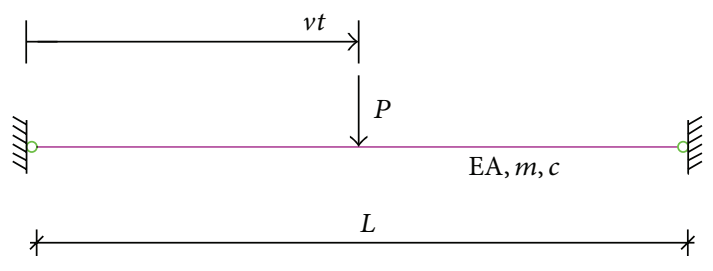

FIGURE 6: Scheme of string moving force.

It is worth pointing out that when the velocity of the mass is bigger than the critical velocity, the string displacement is opposite to the direction of the gravity force which is consistent with our intuition.

\section{The Moving Point Force}

Undamped vibration of the string due to the point force moving with a constant velocity has been considered in the paper [13]. Let us consider now the damped dynamic response of the string due to the point force $P$ moving with a constant velocity (Figure 6 ). In this case, the solutions can be obtained using results of Section 2 by assuming that the length of the segment $a$ tends to zero and taking into account $\lim _{a \rightarrow 0} q a=P=$ const. Rather than that method, it is convenient to consider the differential equation similar to (4) which now has the form

$$
-[w(\xi, T)]^{I I}+c_{0} \dot{w}(\xi, T)+\eta^{2} \ddot{w}(\xi, T)=P_{0} \delta(\xi-T),
$$

where $\delta(\cdot)$ is the Dirac delta and $P_{0}=P L / S$.

The solution for the moving point force, taking initial conditions (6), is a sum of the particular integral $w_{A}=(\xi, T)$ and general integral $w_{S}(\xi, T)$ and has the form

$$
w(\xi, T)=w_{A}(\xi, T)+w_{S}(\xi, T),
$$


where

$$
\begin{aligned}
w_{A}(\xi, T)= & w_{A}^{*}(\xi, T)+w_{A}^{* *}(\xi, T)=2 P_{0}\left(1-\eta^{2}\right) \\
& \times \sum_{n=1}^{\infty} \frac{\sin n \pi T \sin n \pi \xi}{\left[\left(1-\eta^{2}\right)^{2}(n \pi)^{2}+4 \alpha^{2} \eta^{4}\right]}-4 P_{0} \alpha \eta^{2} \\
& \times \sum_{n=1}^{\infty} \frac{\cos n \pi T \sin n \pi \xi}{(n \pi)\left[\left(1-\eta^{2}\right)^{2}(n \pi)^{2}+4 \alpha^{2} \eta^{4}\right]}, \\
w_{S}= & 2 P_{0} e^{-\alpha T} \\
& \times \sum_{n=1}^{\infty} \frac{\left[2 \alpha^{2} \eta^{2}-\left(1-\eta^{2}\right)(n \pi)^{2}\right] \sin \Omega_{n} T \sin n \pi \xi}{\Omega_{n}(n \pi)\left[\left(1-\eta^{2}\right)^{2}(n \pi)^{2}+4 \alpha^{2} \eta^{4}\right]} \\
& +4 P_{0} e^{-\alpha T} \alpha \eta^{2} \\
& \times \sum_{n=1}^{\infty} \frac{\cos \Omega_{n} T \sin n \pi \xi}{(n \pi)\left[\left(1-\eta^{2}\right)^{2}(n \pi)^{2}+4 \alpha^{2} \eta^{4}\right]} .
\end{aligned}
$$

The function $w_{A}(\xi, T)$ which represents aperiodic vibrations can be obtained in the closed form. This function satisfies the nonhomogeneous differential equation (44) but does not satisfy the initial conditions (6). The function

$$
w_{A}^{*}(\xi, T)=2 P_{0} \sum_{n=1}^{\infty} \frac{\left(1-\eta^{2}\right) \sin n \pi T \sin n \pi \xi}{\left[\left(1-\eta^{2}\right)^{2}(n \pi)^{2}+4 \alpha^{2} \eta^{4}\right]}
$$

is a solution of the ordinary differential equation

$$
\frac{d^{2} w_{A}^{*}(\xi, T)}{d \xi^{2}}-\frac{4 \alpha^{2} \eta^{4}}{\left(1-\eta^{2}\right)^{2}} w_{A}^{*}(\xi, T)=-\frac{P_{0}}{1-\eta^{2}} \delta(\xi-T)
$$

for the boundary conditions

$$
w_{A}^{*}(0, T)=w_{A}^{*}(1, T)=0,
$$

and the function

$$
w_{A}^{* *}(\xi, T)=-4 P_{0} \alpha \eta^{2} \sum_{n=1}^{\infty} \frac{\cos n \pi T \sin n \pi \xi}{(n \pi)\left[\left(1-\eta^{2}\right)^{2}(n \pi)^{2}+4 \alpha^{2} \eta^{4}\right]}
$$

is a solution of the ordinary differential equation

$$
\begin{gathered}
\frac{d^{4} w_{A}^{* *}(\xi, T)}{d \xi^{4}}-\frac{4 \alpha^{2} \eta^{4}}{\left(1-\eta^{2}\right)^{2}} \frac{d^{2} w_{A}^{* *}(\xi, T)}{d \xi^{2}} \\
=\frac{2 P_{0} \alpha \eta^{2}}{\left(1-\eta^{2}\right)^{2}} \frac{d \delta(\xi-T)}{d \xi}
\end{gathered}
$$

for the boundary conditions

$$
\begin{gathered}
w_{A}^{*}(0, T)=w_{A}^{*}(1, T)=0, \\
\left.\frac{\partial^{2} w_{A}^{*}(\xi, t)}{\partial \xi^{2}}\right|_{\xi=0}=\left.\frac{\partial^{2} w_{A}^{*}(\xi, t)}{\partial \xi^{2}}\right|_{\xi=1}=0 .
\end{gathered}
$$

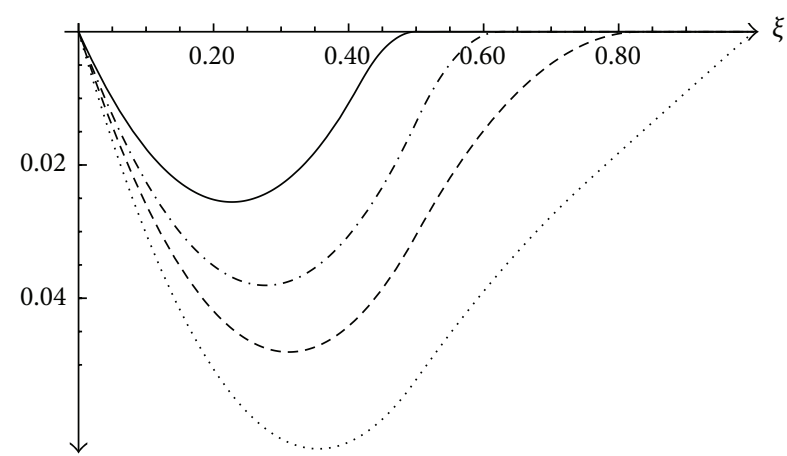

FIGURE 7: Displacement of the string due to uniformly distributed load for $T=0.5$.

After solving (48), (51) using the Laplace transform, we can obtain the function $w_{A}(\xi, T)$ in the closed form instead of a series. These closed solutions have the form

$$
\begin{array}{ll}
w_{A}^{*}(\xi, T)=\frac{P_{0}}{1-\eta^{2}} \frac{\sinh a \xi \sinh a(1-T)}{a \sinh a} & \text { for } \xi \leq T, \\
w_{A}^{*}(\xi, T)=\frac{P_{0}}{1-\eta^{2}} \frac{\sinh a(1-\xi) \sinh a T}{a \sinh a} & \text { for } \xi \geq T,
\end{array}
$$

where $a=2\left(\alpha \eta^{2} /\left(1-\eta^{2}\right)\right)$ and

$$
\begin{aligned}
& w_{A}^{* *}(\xi, T)= \frac{P_{0}}{\left(1-\eta^{2}\right) a} \\
& \times\left[\xi-\frac{\sinh a \xi \cosh a(1-T)}{\sinh a}\right], \quad \text { for } \xi \leq T, \\
& w_{A}^{* *}(\xi, T)= \frac{P_{0}}{\left(1-\eta^{2}\right) a} \\
& \times\left[-(1-\xi)+\frac{\sinh a(1-\xi) \cosh a T}{\sinh a}\right] . \\
& \text { for } \xi \geq T .
\end{aligned}
$$

\section{Some Numerical Results}

In Figures 7 and 8, we present damped displacements of the string for some parameter $\eta=v v_{s}^{-1}$. In Figure 7, we show results in the case of the uniformly distributed moving load and in Figure 8 in the case of a point moving force. In both figures, the dotted line presents the displacement of the string for $\eta=0.4$, dashed line for $\eta=0.6$, the dot-dashed line for $\eta=$ 0.8 , and the solid line for $\eta=1,2$. The damping coefficient has been assumed to be equal to $\alpha=0.03 \pi \eta^{-1}$.

\section{Conclusions}

The dynamics response of a string loaded by a moving load uniformly distributed on an interval or a constant force moving with a constant velocity has been studied. The classical solutions for the transverse displacement of the string have 


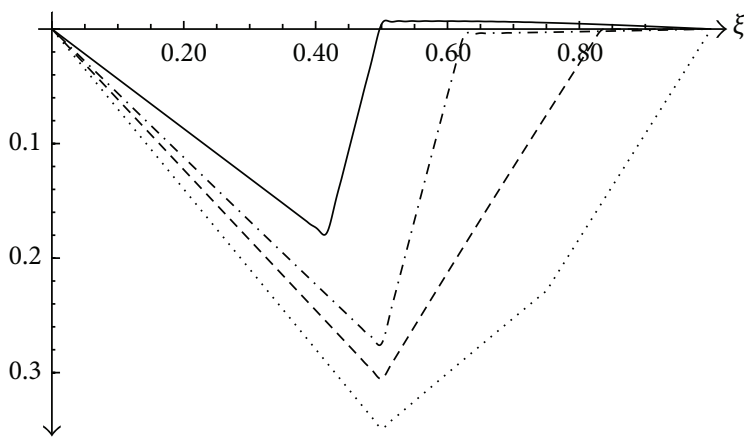

FIGURE 8: Displacement of the string due to a moving point force for $T=0.5$.

the form of sums of infinite series. It has been shown that a part of the solution can be presented in a closed, analytical form. The closed, analytical solutions are derived from the fact that they are not only integrals of partial differential equations but also of some ordinary differential equations. The closed forms of the solutions take different forms whether the velocity of moving load is smaller, equal or larger than the wave velocity of the string. This follows from the fact that in a string wave phenomena may occur. The presented closed solutions have important meaning in the case when we consider the tension force in the string. The closed solutions allow analyzing the vibrations phenomena due to moving loads without performing numerical calculations; see Figure 2. It is worthwhile underlining that presented solutions for the string can be also used in axial and torsional vibration of the bar under moving load (see e.g., [19]). According to the paper [17], these solutions can also be used considering torsional vibrations of multispan bars.

\section{References}

[1] L. Fryba, Vibration of Solids and Structures under Moving Load, Telford, London, UK, 1999.

[2] J. B. Gunda, R. K. Gupta, and R. Ganguli, "Hybrid stiff-stringpolynomial basis functions for vibration analysis of high speed rotating beams," Computers \& Structures, vol. 87, no. 3-4, pp. 254-265, 2009.

[3] J. B. Gunda and R. Ganguli, "Stiff-string basis functions for vibration analysis of high speed rotating beams," Journal of Applied Mechanics, Transactions ASME, vol. 75, no. 2, pp. 0245021-0245025, 2008.

[4] A. V. Kumar and R. Ganguli, "Analogy between rotating EulerBernoulli and Timoshenko beams and stiff strings," Computer Modeling in Engineering \& Science, vol. 88, pp. 443-474, 2012.

[5] C. S. Smith, "Motions of a stretched string carrying a moving mass particle," Journal of Applied Mechanics, Transactions of the ASME, vol. 31, pp. 29-37, 1964.

[6] A. Phylactopoulos and G. G. Adams, "The response of a nonuniformly tensioned circular string to a moving load," Journal of Sound and Vibration, vol. 182, no. 3, pp. 415-426, 1995.

[7] H. A. Dieterman and A. V. Kononov, "Uniform motion of a constant load along a string on an elastically supported membrane," Journal of Sound and Vibration, vol. 208, no. 4, pp. 575-586, 1997.
[8] H. Kruse, K. Popp, and A. V. Metrikine, "Eigenfrequencies of a two-mass oscillator uniformly moving along a string on a viscoelastic foundation," Journal of Sound and Vibration, vol. 218, no. 1, pp. 103-116, 1998.

[9] S. Gavrilov, "Non-stationary problems in dynamics of a string on an elastic foundation subjected to a moving load," Journal of Sound and Vibration, vol. 222, no. 3, pp. 345-361, 1999.

[10] Z. Oniszczuk, "Transverse vibrations of elastically connected double-string complex system-part I: free vibrations," Journal of Sound and Vibration, vol. 232, no. 2, pp. 355-366, 2000.

[11] Z. Oniszczuk, "Transverse vibrations of elastically connected double-string complex system-part II: forced vibrations," Journal of Sound and Vibration, vol. 232, no. 2, pp. 367-386, 2000.

[12] Z. Oniszczuk, "Damped vibration analysis of an elastically connected complex double-string system," Journal of Sound and Vibration, vol. 264, no. 2, pp. 253-271, 2003.

[13] J. Rusin, P. Śniady, and P. Śniady, "Vibrations of double-string complex system under moving forces. Closed solutions," Journal of Sound and Vibration, vol. 330, no. 3, pp. 404-415, 2011.

[14] C. I. Bajer and B. Dyniewicz, "Space-time approach to numerical analysis of a string with a moving mass," International Journal for Numerical Methods in Engineering, vol. 76, no. 10, pp. 1528-1543, 2008.

[15] Z. Kączkowski, "Vibration of a beam under a moving load," Proceedings of Vibration Problems, vol. 4, no. 4, pp. 357-373, 1963.

[16] Z. Reipert, "Vibration of a beam arbitrarily supported on its edges under moving load," Proceedings of Vibration Problems, vol. 2, no. 10, pp. 249-260, 1969.

[17] Z. Reipert, "Vibration of frames under moving load," Archiwum Inżynierii Lądowej, vol. 16, no. 3, pp. 419-447, 1970.

[18] P. Śniady, "Dynamic response of a Timoshenko beam to a moving force," Journal of Applied Mechanics, Transactions of the ASME, vol. 75, pp. 024503-1-00245034, 2008.

[19] D. H. Hodges and G. A. Pierce, Introduction to Structural Dynamics and Aeroelasticity, Cambridge University Press, 2002. 


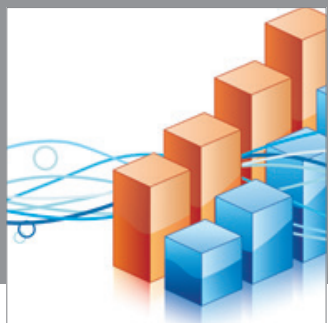

Advances in

Operations Research

mansans

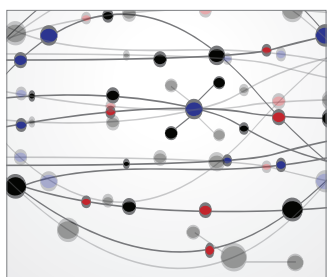

The Scientific World Journal
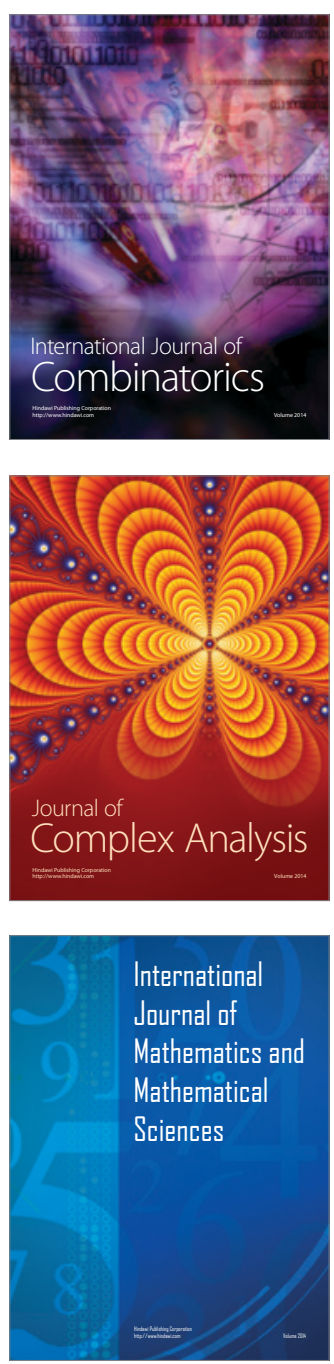
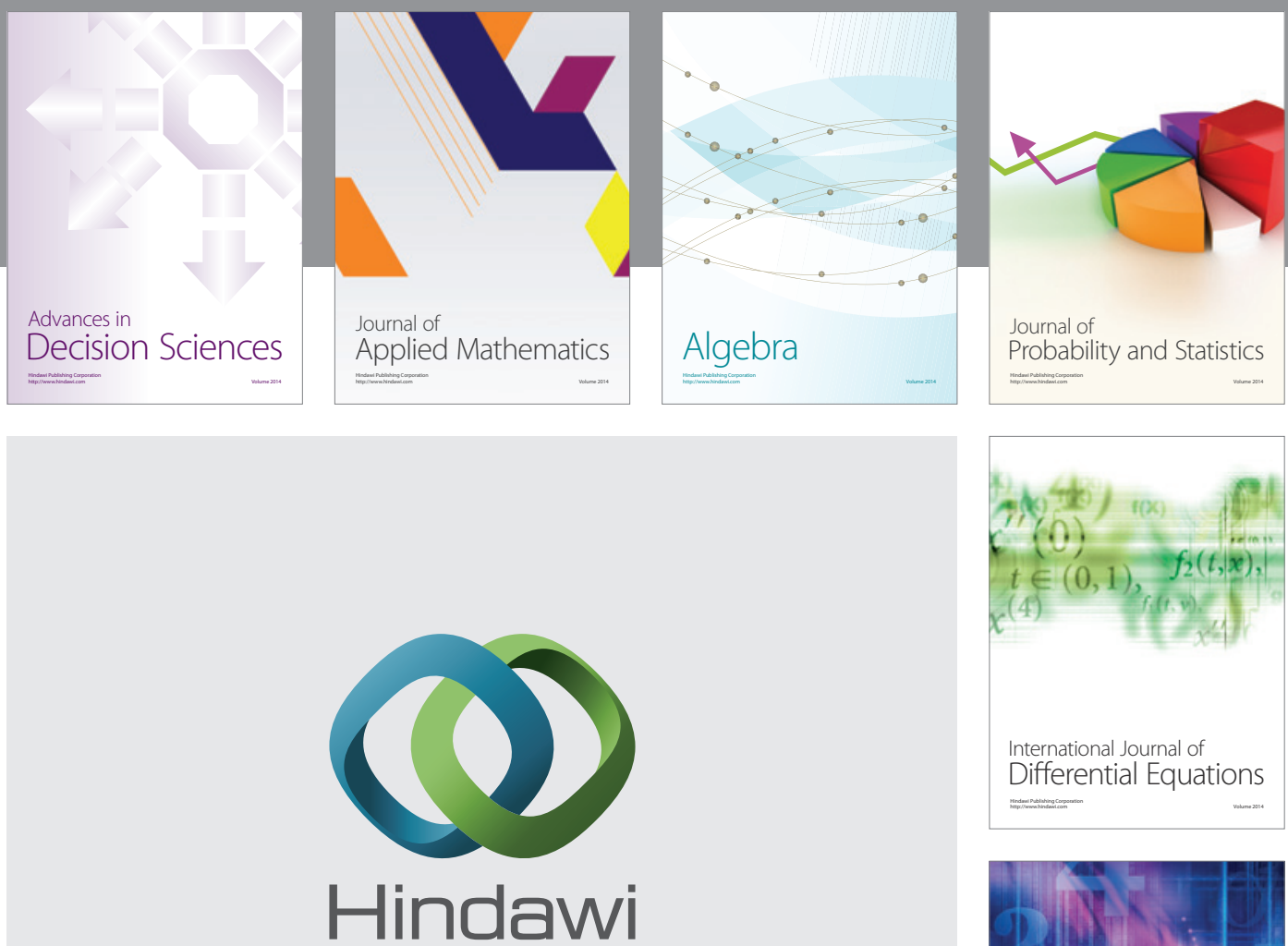

Submit your manuscripts at http://www.hindawi.com
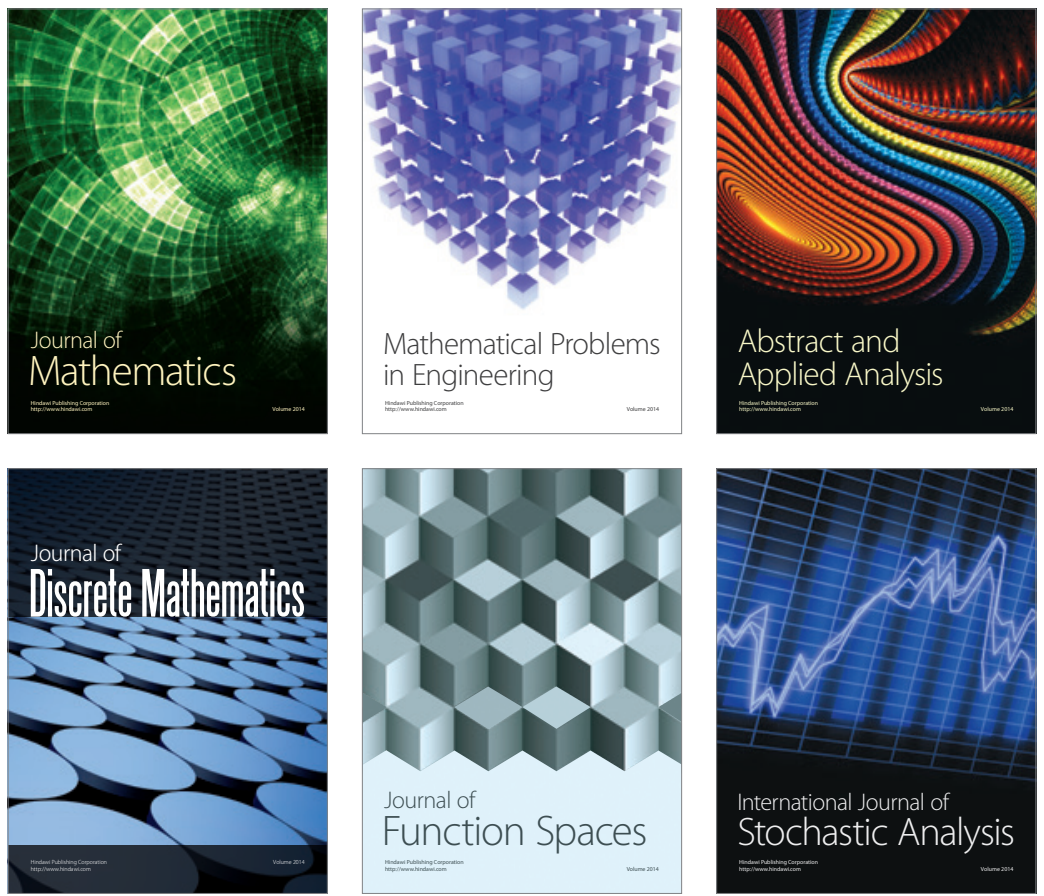

Journal of

Function Spaces

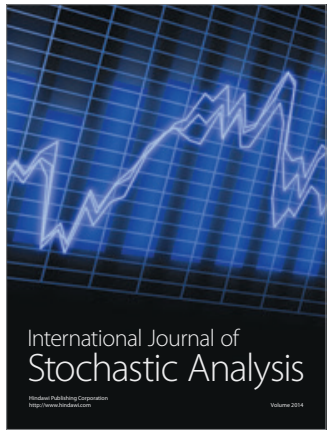

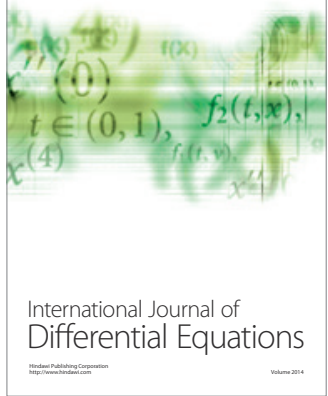
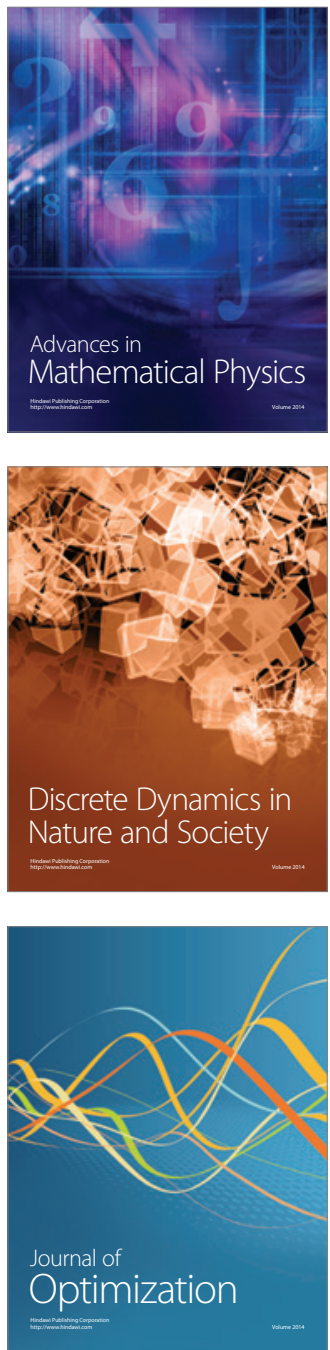